Design Optimization of Vena Cava Filters: An application to dual filtration devices

M. A. Singer, S. L. Wang, D. P. Diachin

December 4, 2009

Journal of Biomechanical Engineering 
This document was prepared as an account of work sponsored by an agency of the United States government. Neither the United States government nor Lawrence Livermore National Security, LLC, nor any of their employees makes any warranty, expressed or implied, or assumes any legal liability or responsibility for the accuracy, completeness, or usefulness of any information, apparatus, product, or process disclosed, or represents that its use would not infringe privately owned rights. Reference herein to any specific commercial product, process, or service by trade name, trademark, manufacturer, or otherwise does not necessarily constitute or imply its endorsement, recommendation, or favoring by the United States government or Lawrence Livermore National Security, LLC. The views and opinions of authors expressed herein do not necessarily state or reflect those of the United States government or Lawrence Livermore National Security, LLC, and shall not be used for advertising or product endorsement purposes. 


\section{Design Optimization of Vena Cava Filters: An application to dual filtration devices}

Corresponding author:

Michael A. Singer

Center for Applied Scientific Computing

Lawrence Livermore National Laboratory

7000 East Avenue, L-422

Livermore, CA 95440

(925) 424-6529 (voice)

(925) 424-2477 (fax)

msinger@llnl.gov

Stephen L. Wang

Division of Vascular and Interventional Radiology

Kaiser Permanente

Santa Clara, CA

Darin P. Diachin

Kanoga Technologies

Livermore, CA 


\title{
Design Optimization of Vena Cava Filters: An application to dual filtration devices
}

\author{
Michael A. Singer ${ }^{a}{ }^{*}$, Stephen L. Wang, ${ }^{b}$ Darin P. Diachin ${ }^{c}$ \\ ${ }^{a}$ Center for Applied Scientific Computing, Lawrence Livermore National \\ Laboratory, Livermore, CA 94551, USA \\ b Division of Vascular and Interventional Radiology, Kaiser Permanente, Santa \\ Clara Medical Center, Santa Clara, CA 95051, USA \\ c Kanoga Technologies, Livermore, CA 94551, USA
}

\begin{abstract}
Background: Pulmonary embolism (PE) is a significant medical problem that results in over 300,000 fatalities per year. A common preventative treatment for PE is the insertion of a metallic filter into the inferior vena cava that traps thrombi before they reach the lungs. The goal of this work is to use methods of mathematical modeling and design optimization to determine the configuration of trapped thrombi that minimizes the hemodynamic disruption. The resulting configuration has implications for constructing an optimally designed vena cava filter.
\end{abstract}

Approach: Computational fluid dynamics is coupled with a nonlinear optimization algorithm to determine the optimal configuration of trapped model thrombus in the inferior vena cava. The location and shape of the thrombus are parameterized, and an objective function, based on wall shear stresses, determines the worthiness of a given configuration. The methods are fully automated and demonstrate the capabilities of a design optimization framework that is broadly applicable.

Results: Changes to thrombus location and shape alter the velocity contours and wall shear stress profiles significantly. For vena cava filters that trap two thrombi simultaneously, the undesirable flow dynamics past one thrombus can be mitigated by leveraging the flow past the other thrombus. Streamlining the shape of thrombus trapped along the cava wall reduces the disruption to the flow, but increases the area exposed to abnormal wall shear stress.

Conclusions: Computer-based design optimization is a useful tool for developing vena cava filters. Characterizing and parameterizing the design requirements and constraints is essential for constructing devices that address clinical complications. In addition, formulating a well-defined objective function that quantifies clinical risks and benefits is needed for designing devices that are clinically viable.

Keywords: CFD, Design optimization, Inferior vena cava, Overset grids, IVC filter 


\section{Introduction}

Deep vein thrombosis (DVT) and pulmonary embolism (PE) are collectively referred to as venous thromboembolism (VTE), which is the third leading cause of cardiovascular related death, after myocardial infarction and stroke [1]. It is estimated that there are more than 900,000 cases of VTE per year in the U.S., and approximately 300,000 individuals die of PE every year [2,3]. PE is the result of deep venous thrombus from the lower extremities that migrate to the lungs. The most common treatment for PE and DVT is anticoagulation therapy (e.g., coumadin, warfarin), which thins the blood and reduces the risk of clotting. In patients whom such therapy is ineffective or contraindicated, however, inferior vena cava (IVC) filters provide an alternative treatment. Usually constructed from nitinol or stainless steel, vena cava filters are metallic devices that are inserted into the IVC through the femoral or jugular vein; the image-guided procedure is usually performed under fluoroscopy. Once deployed in the IVC, the filter is designed to trap large thrombus before they reach the heart and lungs. There are approximately eight IVC filters that are approved for use in the U.S., and approximately 100,000 filters are deployed annually [4].

Despite their widespread use, there is no study in the open literature that seeks to construct an optimally designed vena cava filter based on hemodynamic considerations. In the clinical context, an optimal filter is symmetric, deployable from either the jugular or femoral veins, offers retrievability, has a simple and low profile deployment mechanism, is not prone to tilting, and, perhaps most importantly, is not thrombogenic. Thrombogenicity is correlated with hemodynamic conditions such as abnormal wall shear stress (WSS) and regions of stagnant/recirculating flow $[5,6]$. Consequently, from a flow dynamics viewpoint, an optimally designed filter is one that traps thrombus in a configuration that minimizes the impact of these abnormal flow conditions and therefore minimizes the risk of IVC occlusion.

The present work lays the foundation for a mathematical modeling and optimization framework for designing vena cava filters. In particular, we focus on minimizing the hemodynamic disruptions caused by different locations, sizes, and shapes of model thrombus that may be trapped by a dual filtration vena cava filter, i.e., a filter with two trapping positions. The extent to which thrombi are deformable to the filter geometry is situation-specific, but filter design may enable reshaping of thrombi. We presume that the filter traps one thrombus along the wall of the IVC and a second thrombus in the center of the IVC near the renal inflow; a model of the filter is not included in this

\footnotetext{
* Corresponding author.

Email address: msinger@llnl.gov (Michael A. Singer).
} 
study. This trapping configuration is similar to that of the TrapEase vena cava filter (Cordis, Miami Lakes, FL). By moving and re-shaping the clots in a virtual computer simulation environment without the physical constraints imposed by a particular filter design, the optimization procedure strives to minimize the disruption caused by the thrombus that is trapped along the cava wall. Once an optimal configuration of the model thrombus is achieved, an IVC filter can be constructed that traps thrombus in the optimal arrangement; design of such a device is not considered here. In taking the modeling approach described above, the configuration of the trapped thrombus serves as the fundamental criterion for evaluating filter design. Indeed, this metric is appropriate given that trapped thrombus generally disturb the flow more than a conventionally designed filter that is not occluded.

Previous studies have also combined flow modeling tools and design optimization software. In the work of Marsden et al. [7], for example, a derivativefree optimization framework for cardiovascular flows was constructed, and the methodology was applied to three shape optimization problems: stenosis, vessel bifurcation, and anastomosis. Computational fluid dynamics and optimization methods are combined in the free-form haptic anatomy editing tool, SURGEM, which evaluates modifications of patient-specific anatomies for surgical planning [8]. Design optimization techniques have also been applied to medical devices, most notably stents (e.g., [9-11] among others). In all of these studies, one of the primary motivations is to minimize disruption to otherwise normal physiological flow. The objective of this work is similar, and it represents the first application of numerical optimization methods to vena cava filters.

\section{Methods}

The computer modeling techniques used to perform the design optimization study are discussed in this section. In particular, the computational representation of the physiological vasculature is discussed, as is the representation of model thrombus. The numerical techniques used to compute the hemodynamics and perform the design optimization are also explained.

\subsection{Computational model}

The computational model is a two-dimensional representation of the physiology in and near the IVC (Fig. 1). In particular, the anatomy considered includes: the IVC, which is the focus of this study; the common iliac veins, which provide inflow from the lower extremities (e.g., legs); and the renal veins, 

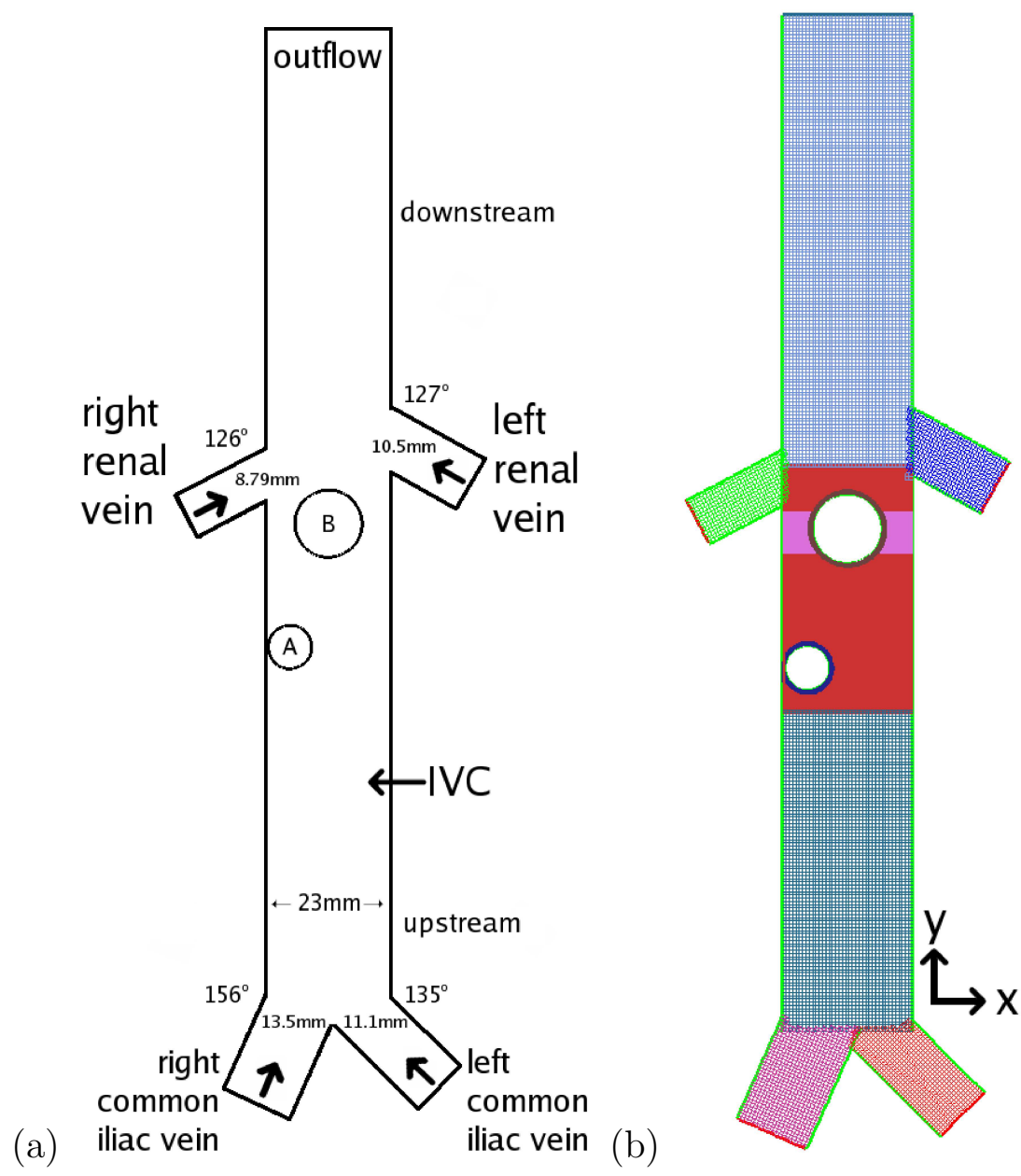

Fig. 1. Two-dimensional computational model: (a) schematic of the flow configuration; (b) ten overlapping grids used to discretize the geometry, including the higher resolution grids (red and violet) near the two model thrombus.

which provide inflow from the kidneys. All vessels are taken to be straight and rigid (e.g., [12-16]), and the IVC has an inner diameter of $23 \mathrm{~mm}$, per the average cava diameter in [17]. The diameters and entry angles of the common iliac and renal veins into the IVC, as noted in Fig. 1, are computed from abdominal CT scans of 24 adult patients (12 males, 12 females; average age 55 , age range 16-89) with no known renal disease or anatomical variants. In particular, the diameters of the common iliac and renal veins are measured from IV contrast enhanced $2.5 \mathrm{~mm}$ slice thickness axial CT images; the entry angles are measured using PACS software from IV contrast enhanced $5 \mathrm{~mm}$ slice thickness coronal CT images reformatted from the axial source images. The measurements used to construct the model are the average values from all 24 patients. All patient-specific anatomic information was obtained at Kaiser Permanente and was given exemption status after meeting our Institutional Review Board and federal regulatory criteria for exemption.

Thrombus are modeled as rigid objects with two-dimensional areas corre- 
sponding to clot volumes of $0.5 \mathrm{~mL}, 1 \mathrm{~mL}$, and $2 \mathrm{~mL}$, which are volumes representative of those studied in-vitro by Wang et al. [18]. The radius of a $1 \mathrm{~mL}$ clot, for example, is the radius of a circle that represents the $1 \mathrm{~mL}$ clot in the two-dimensional model. Figure 1 shows a representative configuration of the multi-clot system being considered: clot A lies along the wall of the cava in an upstream trapping position, and clot B lies in the center of the cava in a downstream trapping position near the renal vein inflow. This dual filtration trapping configuration is representative of, for example, the TrapEase vena cava filter. In this design optimization study, clot A is free to move upstream and downstream along the wall of the vessel; hence, the axial position of clot A is a design parameter. In addition, the shape of clot $\mathrm{A}$, as defined by its aspect ratio, is also a design parameter, though the area of clot $\mathrm{A}$ is held constant. Note that in all cases, clot A is constrained to lie along the vessel wall, which is a representative trapping position for dual filtration filters (e.g., TrapEase) and filters that are severely tilted (e.g., Günther Celect [Cook, Bloomington, IN], Recovery [Bard Peripheral Vascular, Tempe, AZ]) [15]. Our optimization framework does not inherently impose this restriction, however, and allowing the clot to separate from the cava wall introduces another design parameter, i.e., the x-coordinate. In all cases, clot B remains stationary and is positioned in relation to the renal inflow per the study of Wang and Singer [14].

Blood is modeled as a homogeneous, incompressible, Newtonian fluid with density $\rho=1040 \mathrm{~kg} / \mathrm{m}^{3}$ and dynamic viscosity $\mu=2.57 \times 10^{-3} \mathrm{~kg} /(\mathrm{ms})$. As discussed in [16], the Newtonian approximation is appropriate for the present flow regime. The conservation equations that describe the flow are the incompressible Navier-Stokes (NS) equations, and the flow is taken to be steady-state. Flow through the IVC is characterized by the Reynolds number, $\operatorname{Re}=\rho U D / \mu$, where $\rho, U, D, \mu$ are the density, mean inlet velocity of flow into the IVC, diameter of the IVC, and dynamic viscosity, respectively.

Use of a two-dimensional model significantly reduces the computational cost of the design optimization process in comparison to a three-dimensional representation of the geometry. While it is recognized that the physiological flow is three-dimensional, the computational expense of using a three-dimensional model is appreciably more than the two-dimensional case. Nonetheless, important hemodynamic features that are critical for evaluating filter performance (e.g., stagnant/recirculating flow, WSS) are captured in the two-dimensional model, and the model includes anatomical features such as asymmetric common iliac and renal vein attachment angles and inflow velocities. 


\subsection{Numerical methods}

The design optimization framework combines methods of computational fluid dynamics (CFD) and nonlinear optimization: CFD is used to compute the flow field, and numerical optimization is used to determine the optimal design configuration. The goal of the optimization procedure is to minimize the value of an objective function (OF) that quantifies the clinical worthiness of a given clot configuration (i.e., location and shape). Details of the OF, whose meaningful formulation is crucial for obtaining clinically relevant results, is discussed in section 2.3.

The CFD and optimization procedures are executed with separate computer codes, and the two codes are coupled in a fully automated fashion using Python scripts. Hence, the computations proceed as follows: the flow field is computed (using CFD) for a given location and shape of clot A; using the computed flow field, the $\mathrm{OF}$ is evaluated; based on the current and previous values of the $\mathrm{OF}$, the optimization procedure determines how to move or reshape the clot in an attempt to reduce the value of the OF; the location/shape of clot A are modified, and a new computational grid is generated; the flow field is recomputed. Multiple computer processors are used concurrently to accelerate convergence of the overall optimization algorithm. Details of the CFD and optimization methods are described below.

\subsubsection{Computational fluid dynamics}

The flow field is determined by solving the incompressible NS equations using the Overture software framework. As discussed elsewhere (e.g., [19]), Overture uses finite difference approximations on overset grids to discretize the equations on a collection of overlapping, curvilinear grids. This work uses second-order accurate spatial approximations, and the steady-state solution is computed using a pressure-based equation solver [20,21]. Artificial diffusion, based on second- and fourth-order undivided differences, is also used. The diffusion terms are added to the momentum equation to improve stability and do not affect the order of accuracy of the method. A no-slip condition is imposed at all solid walls, and parabolic inlet velocity profiles are prescribed at the inflow of the common iliac and renal veins. The outlet flow condition, imposed at the outflow of the IVC, is a mixed (Robin) condition on the pressure and an extrapolation on the velocity.

The computational domain, shown in Fig. 1a, is discretized using 10 overlapping, Cartesian grids as shown in Fig. 1b. High resolution grids cover the region between clots $\mathrm{A}$ and $\mathrm{B}$, which contains stagnant and recirculating flow due to vorticity introduced by clot A. Coarser grids are used upstream of clot 
A, far downstream of clot B, and in the common iliac and renal veins. Note that as clot A moves and reshapes during the search for the optimal solution, the area occupied by the high resolution grid changes, and therefore the total number of grid points also changes: the computational expense increases as clot A moves upstream.

\subsubsection{Numerical optimization}

The optimization procedure solves the following nonlinear problem with bound constraints

$$
\min \{F(\mathbf{d}): \mathbf{l} \leq \mathbf{d} \leq \mathbf{u}\}
$$

where $F: \mathbb{R}^{p} \rightarrow \mathbb{R}$ is the $\mathrm{OF}$, and $\mathbf{d} \in \mathbb{R}^{p}$ is a vector of $p$ design parameters. For the present study, $\mathbf{d}$ contains the axial location of clot A and, if appropriate, the aspect ratio of the clot; the vectors $\mathbf{l} \in \mathbb{R}^{p}$ and $\mathbf{u} \in \mathbb{R}^{p}$ contain the corresponding lower and upper bounds on the spatial coordinate and the aspect ratio. Details on the formulation and interpretation of $F$ are discussed in section 2.3 .

The minimization problem (1) is solved with APPSPACK 5.0.1 [22,23], which is an open-source, asynchronous, parallel, derivative-free optimization code. APPSPACK uses a pattern search method to obtain the optimal solution by iteratively searching for updates to the current design (which is parameterized by the quantities in $\mathbf{d}$ ) that are within a positive spanning set of $\mathbb{R}^{p}$. That is, the $\mathrm{OF}$ is computed at predetermined step lengths in each direction of the spanning set. If no reduction to the OF is identified, the step length is reduced and the search is repeated. Under certain conditions, APPSPACK is guaranteed to converge to a local minimum, and for some global optimization problems it has demonstrated to perform competitively with global optimization methods while requiring fewer function evaluations [24].

APPSPACK is well-suited for the design optimization study considered here. Asynchronous execution allows it to perform efficiently on heterogeneous computer networks such as the combination of Pentium and quad-core machines used for this work. It is also efficient in parallel when OF computation times vary considerably depending on the parameter values. For this study, the time required to compute the $\mathrm{OF}$ is strongly dependent on the design parameters; as clot A moves upstream, the number of grid points and the area occupied by stagnant/recirculating flow increases, which can increase the time required to evaluate the OF by a factor of five. Finally, the OF (described below) is a function of WSS, which may be noisy for a number of reasons, including its dependence on velocity gradients. Hence, the derivative-free methodology of APPSPACK avoids reliance on gradients of the OF, which may be inaccurate. 


\subsection{Design optimization criteria}

The objective function provides the specification of optimal design. Consequently, the formulation of an objective function that incorporates medically relevant design criteria is essential for constructing medical devices that are clinically viable. Wall shear stress is a measure of the velocity gradient along the wall of a vessel, and regions of abnormal WSS are correlated with an elevated risk of thrombosis [5,6,25-28]. As a result, medical devices that induce minimal disruption to otherwise normal WSS profiles may reduce the risk of thrombosis caused by the device.

Building on the demonstrated importance of WSS, the OF used here is the following

$$
\begin{aligned}
F(\mathbf{d})= & \sqrt{\sum_{j=1}^{N_{R}}\left(W_{n}\left(R, y_{j} ; \mathbf{d}\right)-W_{b}\left(R, y_{j}\right)\right)^{2}} \\
& +\sqrt{\sum_{j=1}^{N_{L}}\left(W_{n}\left(L, y_{j} ; \mathbf{d}\right)-W_{b}\left(L, y_{j}\right)\right)^{2}}
\end{aligned}
$$

where $W_{n}\left(R, y_{j} ; \mathbf{d}\right)$ and $W_{n}\left(L, y_{j} ; \mathbf{d}\right)$ are the wall shear stresses along the right and left cava wall, respectively. The subscript $n$ denotes the WSS of a new design configuration, where the location and shape of the upstream thrombus ( $\operatorname{clot} \mathrm{A})$ are parameterized by the design variables in $\mathbf{d}$. The wall shear stresses are evaluated at $N_{R}$ or $N_{L}$ discrete y-coordinates denoted $y_{j}$, where $N_{R}$ and $N_{L}$ are the number of grid points along the right and left wall, respectively. The baseline WSS profiles along the right and left walls of the IVC, denoted $W_{b}\left(R, y_{j}\right)$ and $W_{b}\left(L, y_{j}\right)$, are independent of $\mathbf{d}$.

The dual filtration configurations considered here presume that the downstream thrombus, clot B, is located in the center of the IVC and remains stationary. Hence, the goal of the present study is to determine the location and shape of the upstream thrombus, clot A, that minimizes the disruption to the WSS caused by the upstream thrombus. Therefore, the baseline WSS is that of the single thrombus configuration with clot B in the middle of the IVC. Note that selection of this baseline WSS is distinctly different than choosing the baseline WSS to be that of the unoccluded IVC. In the latter case, the goal of the optimization procedure would be to mitigate the negative flow effects of clot B by leveraging the flow disruption caused by clot A. This flow scenario has little clinical relevance to dual filtration filters because thrombus trapped near the center of the vessel are, in some sense, trapped in the ideal location - far from the cava walls. In contrast, thrombus trapped along the wall are known to induce regions of stagnant/recirculating flow along the vessel wall 
that may be thrombogenic $[13-15,25]$

Based on the design optimization criteria described above, it is reasonable to question the clinical value of dual filtration IVC filters that trap thrombus along the cava wall: If the optimal WSS of a dual filtration device is that of a single filtration device, why design dual filtration devices? But, the potential benefits of vena cava filters that trap thrombus along the wall and do not elevate the risk of thrombosis are numerous. Allowing filter designers the flexibility to consider such designs broadens the design space for constructing filters that are symmetric, less prone to tilting, non-thrombogenic, low profile, easily deployed and retrieved, and clinically effective.

\section{Results: spatial convergence and verification}

The two-dimensional, incompressible NS equations are solved to determine the two components of the velocity and the pressure. In all of the figures below, the flow is bottom-to-top, and all velocities are normalized by the average velocity, in the y-direction, of unoccluded flow into the vena cava (e.g., [13-16]). Similarly, the wall shear stresses are normalized by the value in the unoccluded IVC. Both the velocity and WSS used for normalization are obtained by running a simulation with no thrombus. Note that the values used for normalization do not impact the optimization procedure.

The computational model requires the specification of flow velocities ( $\mathrm{x}$ - and y-components) into the common iliac and renal veins. The flow speeds into the common iliac veins are adjusted so that the flow rate into the IVC is $1 \mathrm{~L} / \mathrm{min}$, which is within the range of $1.2 \pm 0.5 \mathrm{~L} / \mathrm{min}$ reported in [30] for infrarenal flow at rest. This flow rate corresponds to a Reynolds number of approximately 370 , which is consistent with the flow conditions used elsewhere (e.g., $[14,15,29])$. Note that the components of the parabolic inflow velocity profiles are computed from the speed and the entry angle of the common iliac vein into the IVC (see Fig. 1a).

Inflow velocities for the right and left renal veins are computed similarly, and the peak flow speeds are $10.1 \mathrm{~cm} / \mathrm{s}(0.18 \mathrm{~L} / \mathrm{min})$ and $10.9 \mathrm{~cm} / \mathrm{s}(0.28 \mathrm{~L} / \mathrm{min})$, respectively. Note that these inflow speeds are one-quarter of those used in [14]. These lower, yet physiologically relevant, renal inflow conditions give a supraceliac flow rate of approximately $1.46 \mathrm{~L} / \mathrm{min}$, which is nearly in the range reported in [30] $(2.0 \pm 0.5 \mathrm{~L} / \mathrm{min})$ for supraceliac flow at rest. These renal inflow conditions are chosen to maximize the potential impact of clot configuration on the WSS downstream of the renal inflow; otherwise, flow through the cava downstream of the renal veins is dictated entirely by the high speed renal inflow. Hence, the low speed renal inflow conditions represent a "worst case" 
condition in the sense that filter design will have the largest impact on WSS throughout the entire IVC.

\subsection{Spatial convergence}

Sufficient spatial resolution, which is especially important because the OF depends on the WSS, is ensured by performing a grid refinement study. The representative thrombus configuration used for the refinement study contains a $0.5 \mathrm{~mL}$ upstream thrombus and a $1 \mathrm{~mL}$ downstream thrombus; the configuration and the corresponding velocity contours are shown in Fig. 2a. Four grid resolutions, low (107K grid points), medium (162K grid points), high (256K grid points), and ultra (406K grid points), are tested, and each successively higher resolution grid contains approximately $50 \%$ more total grid points. Grid refinement is nearly uniform in space (i.e., all 10 grids shown in Fig. 1b are refined by approximately the same amount), which increases the number of grid points in the viscous boundary layers near the thrombi and the walls of the vessels.

(a)

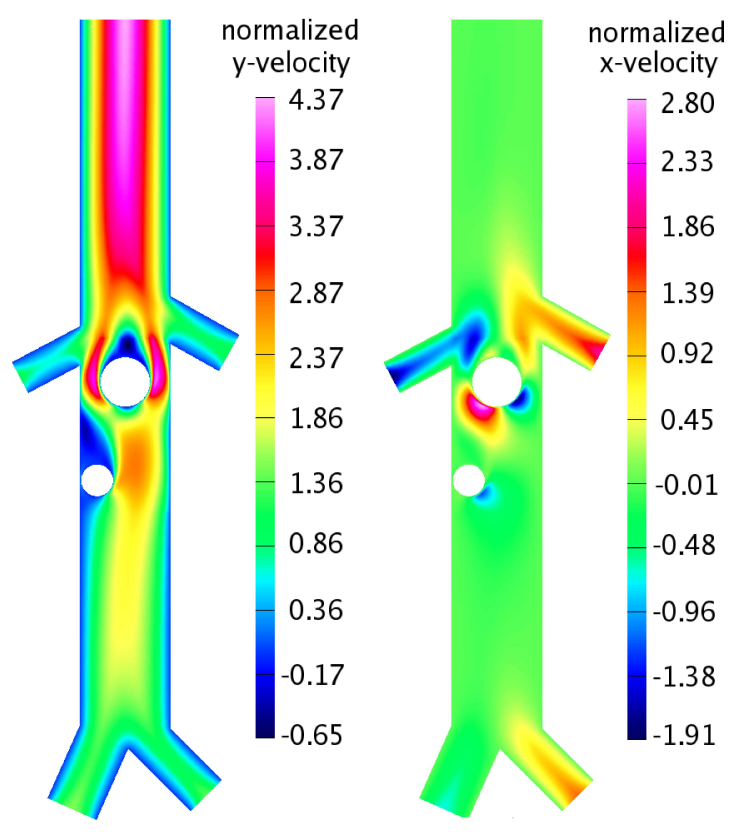

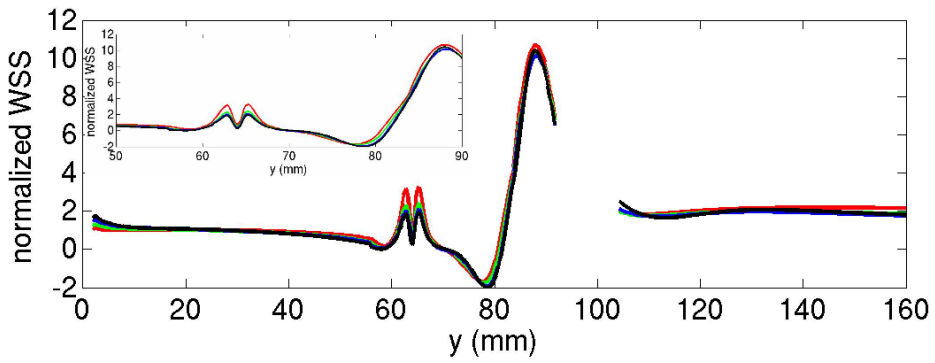

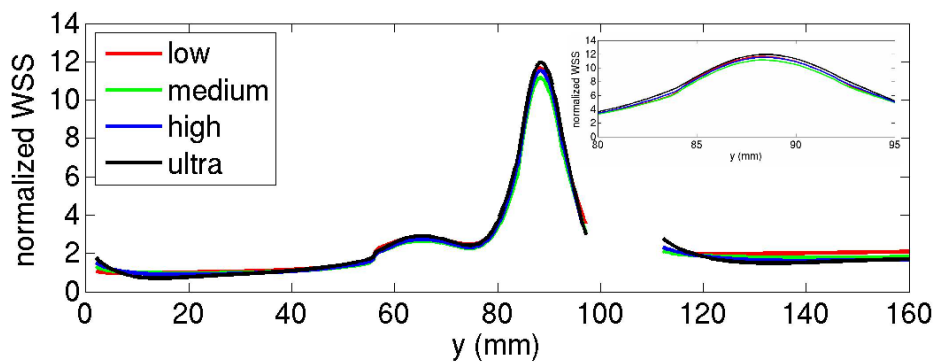

(b)

Fig. 2. Results of the grid refinement study: (a) contour plots of the normalized yand x-components of velocity using the medium grid resolution; (b) convergence of the normalized WSS along the right (top) and left (bottom) walls of the IVC. Gaps in the WSS profiles indicate the locations of renal inflow.

Figure $2 \mathrm{~b}$ shows the normalized wall shear stresses along the cava walls with the different grid resolutions. As demonstrated, the WSS converges monotonically with grid resolution, and the greatest impact of insufficient resolution is near the thrombi and immediately downstream of the renal inflow, especially 
along the right wall of the cava. These regions of cava flow contain stagnation/recirculation zones or large velocity gradients that require sufficient grid resolution to capture completely. As a result, high resolution, overlapping grids are inserted into these areas as demonstrated in Fig. 1b. Concentrating fine grids only in areas with complex flow dynamics reduces the overall number of grid points required to resolve the flow and compute accurate wall shear stresses. Minimizing the computational expense while also maximizing accuracy is especially important for design optimization problems because the flow field often needs to be recomputed each time the OF is evaluated.

Table 1 shows the $L_{2}$ and $L_{\infty}$ errors in the wall shear stress for the low, medium, and high resolution grids along the right and left walls of the cava; the "exact" solution is obtained from the ultra resolution grid. As noted, increasing the grid resolution by a factor of 2.5 (medium to ultra) reduces the error of the WSS by $7-9 \%$. This slight reduction in error is not worth the additional computational expense; hence, the medium grid resolution is used for subsequent studies.

Table 1

\begin{tabular}{|c|c|c|c|}
\hline resolution & grid points & $\mathrm{L}_{2}$ error (R/L WSS) & $\mathrm{L}_{\infty}$ error (R/L WSS) \\
\hline \hline low & $107 \mathrm{~K}$ & $0.148 / 0.0906$ & $0.129 / 0.0639$ \\
\hline medium & $162 \mathrm{~K}$ & $0.0734 / 0.0889$ & $0.0698 / 0.0708$ \\
\hline high & $256 \mathrm{~K}$ & $0.0395 / 0.0502$ & $0.0392 / 0.0374$ \\
\hline ultra & $406 \mathrm{~K}$ & - & - \\
\hline
\end{tabular}

Spatial convergence of the error in the wall shear stress along the right and left walls of the IVC. The solutions obtained using the ultra grid resolution are taken to be the exact solutions. All of the results below use the medium grid resolution.

\subsection{Verification of the CFD/optimization framework}

Verification is the process of ensuring that a computational framework is implemented properly and produces accurate solutions. In contrast, validation ensures that a computational modeling procedure yields results that agree with experimental data. See [31] for a complete discussion of verification and validation for CFD. To verify the correctness of the CFD and optimization capabilities described above, the solutions to three test problems are computed.

First, the steady-state flow past a $1 \mathrm{~mL}$ thrombus, which is horizontally centered in the IVC, is computed (Fig. 3d). The solution to this problem is referred to as the baseline solution and serves as the exact solution to the three test problems used for verification. The location and shape of the thrombus is then perturbed from its baseline location and shape, and this altered configuration serves as the initial condition for the CFD/optimization algorithm. 
The goal of the optimization procedure is then to determine the location and shape of the thrombus that minimizes the OF; $W_{b}$ in the OF, equation (3), is the WSS for the baseline configuration. That is, the WSS of the perturbed configuration is compared to that of the baseline configuration, and the design parameters (i.e., location and shape of the thrombus) are adjusted to minimize the difference.

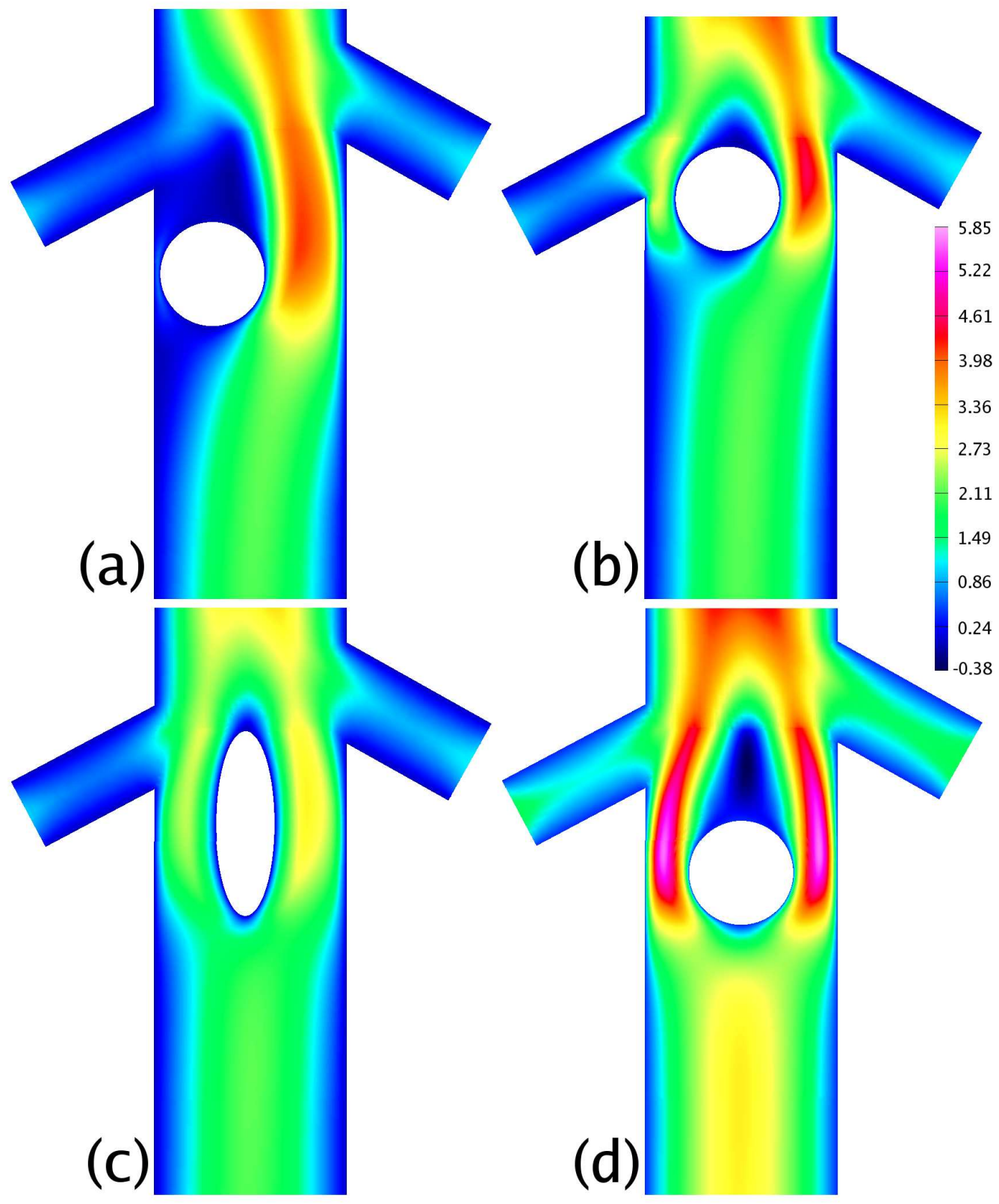

Fig. 3. Normalized contours of the axial velocity for the initial and final configurations of the verification test cases: (a) one parameter problem, (b) two parameter problem, (c) three parameter problem, (d) baseline solution.

The three perturbed configurations are shown in Figs.3a, 3b, and 3c; the corresponding parameter ranges are shown in Table 2. In Fig. 3a, the horizontal 
location of the thrombus is perturbed, and the single design parameter is the $\mathrm{x}$-coordinate of the thrombus. In Fig. 3b, both the $\mathrm{x}$ - and $\mathrm{y}$-coordinates are perturbed, which results in a two parameter design optimization problem. Finally, Fig. 3c shows the initial conditions for a three parameter optimization problem: the $\mathrm{x}$ - and $\mathrm{y}$-coordinates and the aspect ratio of the thrombus are perturbed. Fig. 4 shows the corresponding WSS profiles for the initial and baseline configurations.

As seen in Table 2, the final solutions produced by the CFD/optimization framework are nearly identical to the baseline configuration. As a result, the implementation of our methods is verified. As expected, the number of $\mathrm{OF}$ evaluations increases with the number of design parameters. Validation is not addressed here due to a lack of experimental data.
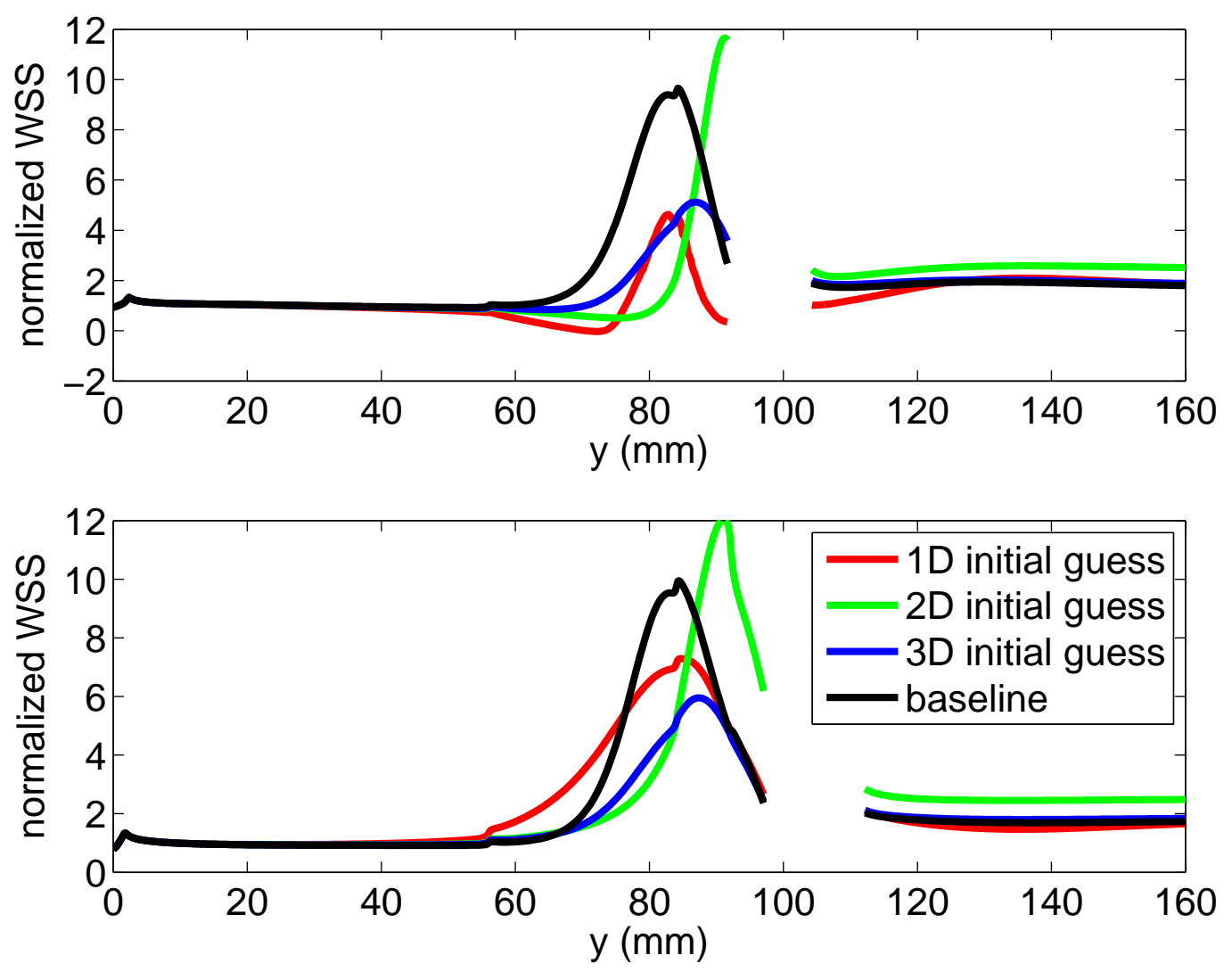

Fig. 4. Normalized WSS profiles for the starting and baseline configurations of the verification test problems: top figure is WSS along the right cava wall; bottom figure is WSS along the left cava wall. Gaps in the WSS profiles indicate the locations of renal inflow. 


\begin{tabular}{|c|c|c|c|c|c|}
\hline params & {$[x$-range $] / x_{\mathrm{opt}}(\mathrm{mm})$} & {$[y$-range $] / y_{\mathrm{opt}}(\mathrm{mm})$} & {$[a$-range $] / a_{\mathrm{opt}}$} & value of OF & OF evals \\
\hline \hline 1 & {$[-5,5] / 0.0$} & - & - & $8.939 \times 10^{-3}$ & 22 \\
\hline 2 & {$[-4.4,4.4] / 0.0$} & {$[72.75,88.75] / 82.75$} & - & $8.939 \times 10^{-3}$ & 48 \\
\hline 3 & {$[-1.6,1.6] / 0.0$} & {$[76.75,90.75] / 82.73$} & {$[0.55,6.66] / 0.997$} & 2.077 & 155 \\
\hline
\end{tabular}

Table 2

Optimization parameters and results for the verification test problems, where $a$ is the aspect ratio of clot $\mathrm{A}$. The exact solution is $\left(x_{\mathrm{opt}}, y_{\mathrm{opt}}, a_{\mathrm{opt}}\right)=(0.0,82.75,1.0)$.

\section{Results: design optimization studies}

This section describes results from the one and two parameter design optimization studies. In section 4.1, the single design variable is the location of the upstream thrombus; the clot is confined to the right wall of the cava. In section 4.2 , the location and shape of the upstream thrombus are design parameters, and the clot is again confined to the right wall of the cava. The flow conditions are the same as described in section 3.

\subsection{One parameter problem: Variable location of the upstream thrombus}

The y-coordinate of the upstream thrombus is the single design parameter in the one parameter optimization problem; the clot remains confined to the cava wall. The optimization procedure then seeks to minimize the OF by varying the axial location of clot A. Normalized contours of the axial velocity component for the baseline and optimal thrombus configurations are shown in Figs 5a and 5b. In each set of figures, the baseline case, from which the baseline WSS is computed, is on the left. As illustrated, there are regions of stagnant/recirculating flow downstream of clot B, and the area occupied by low velocity flow increases with the size of clot B. In addition, low velocity flow is observed upstream and downstream of clot A, and the area of disrupted flow increases with the size of the upstream thrombus. As the size of clot A increases, flow is directed toward the left wall of the cava, which increases the velocity of flow through the narrow passage between clot B and the left wall of the cava. At the same time, a high speed jet develops that drives transverse flow (not shown in the contours of axial velocity) between the narrow passage that separates clots $\mathrm{A}$ and $\mathrm{B}$. This jet adds momentum to the wake of clot A and reduces the size of the stagnation/recirculation zone downstream of clot A.

Figures $6 \mathrm{a}$ and $6 \mathrm{~b}$ show the normalized WSS profiles along the right (top) and left (bottom) walls of the IVC for the optimal configurations. The peak WSS increases with the size of clot B and occurs in the narrow passage that 

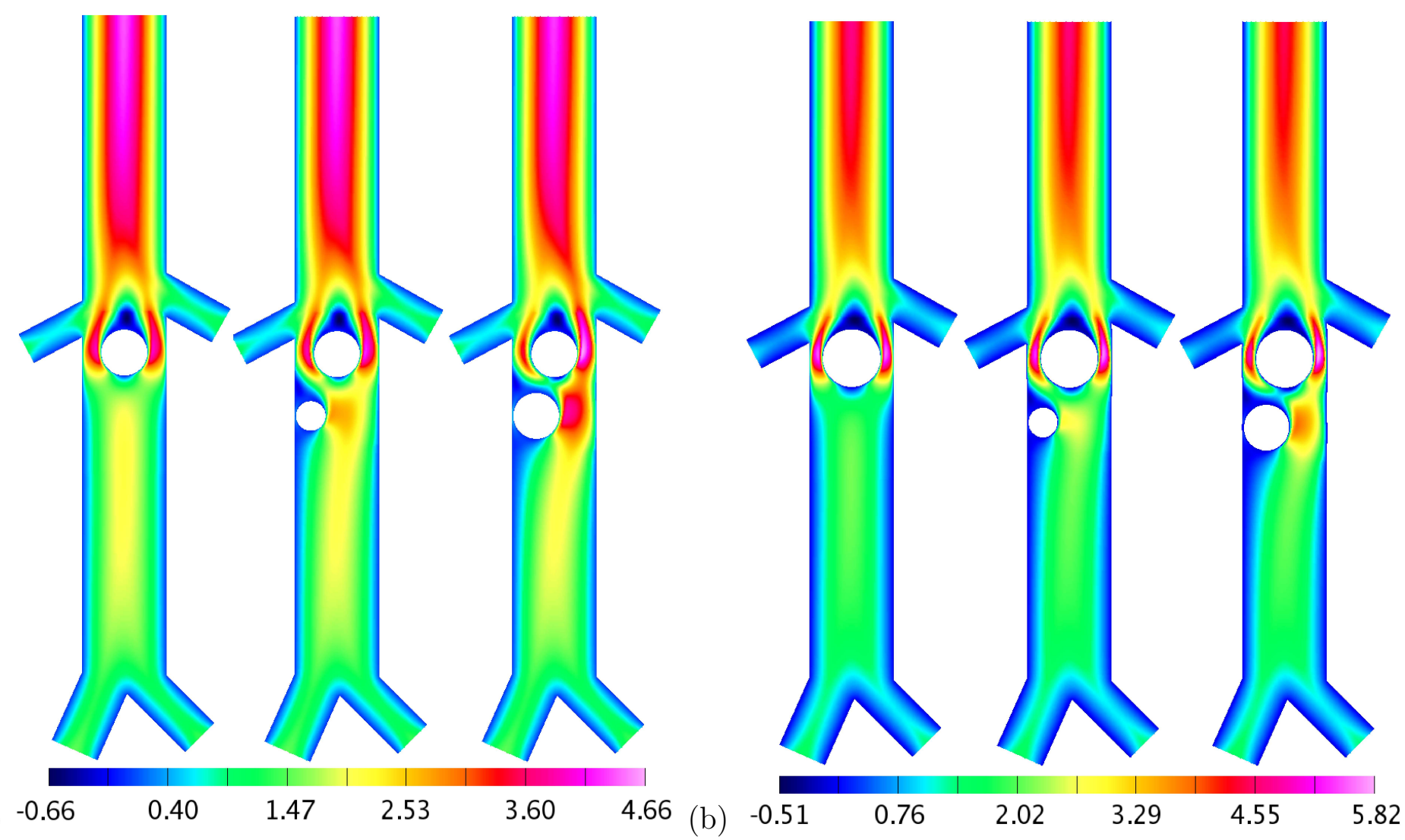

Fig. 5. Contours of the normalized y-component of velocity for the baseline and optimal configurations with one design parameter: (a) no (left), 0.5mL (center), and $1 \mathrm{~mL}$ (right) thrombus upstream and $1 \mathrm{~mL}$ thrombus downstream; (b) no (left), $0.5 \mathrm{~mL}$ (center), and $1 \mathrm{~mL}$ (right) thrombus upstream and $2 \mathrm{~mL}$ thrombus downstream.

separates clot B from the left cava wall. As indicated, the wall shear stresses along the right wall, both upstream and downstream of clot $\mathrm{A}$, are negative, which further indicates regions of flow reversal. The fraction of vessel occupied by abnormal WSS, where normal is defined by the baseline case, is similar in all cases, though the amplitude of the abnormalities along the left cava wall increases with the size of clot A. The locations of renal inflow are denoted by gaps in the WSS profiles (since the WSS is not defined in these areas), and in all cases the WSS profiles downstream of the renal inflow are identical. This latter observation further indicates the dominance of renal inflow in determining the flow characteristics downstream of the renal veins, and demonstrates that WSS downstream of renal inflow is nearly independent of thrombus configuration upstream of the renal inflow. For cases with the $2 \mathrm{~mL}$ downstream thrombus (Fig. 6b), the locations of the peak wall shear stresses along both cava walls are well-aligned with the peak of the baseline configuration. Indeed, different locations of the peak wall shear stresses increase the value of the OF, so alignment of the peaks help drive the optimal location of the upstream thrombus. 

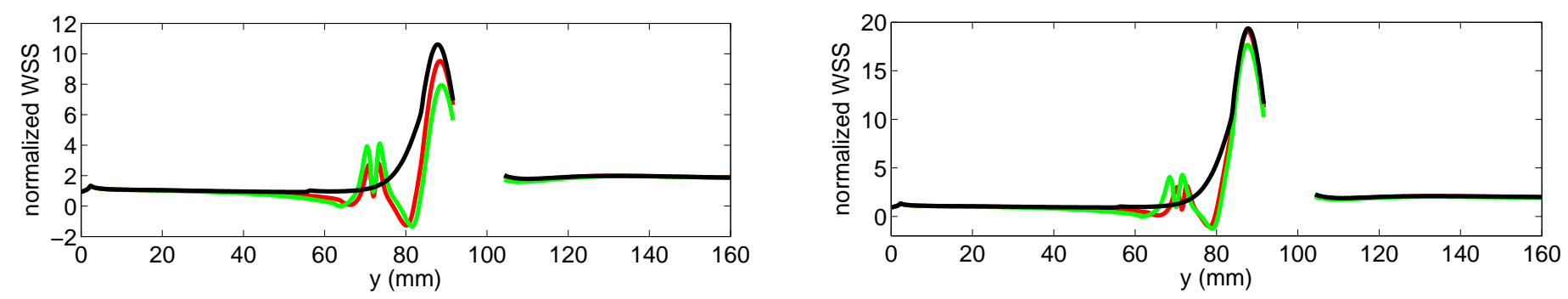

(a)
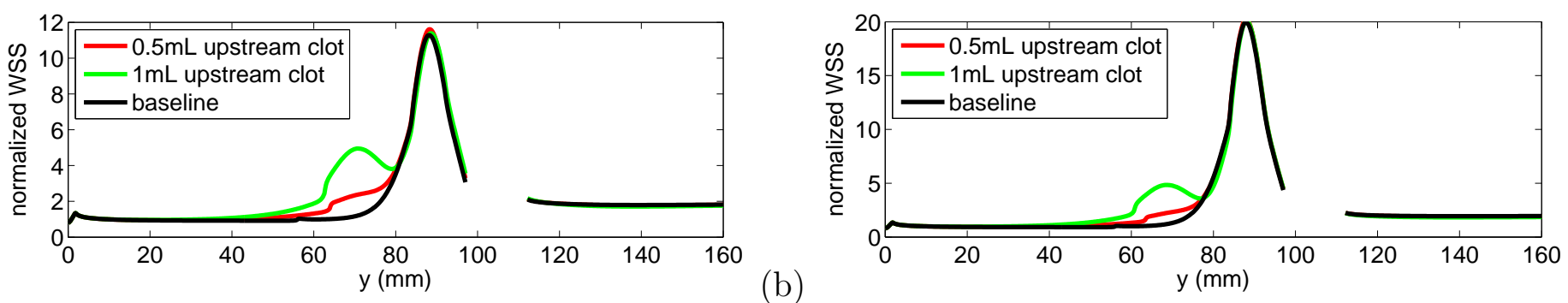

Fig. 6. Normalized WSS profiles for the optimal configurations with one design parameter: (a) $1 \mathrm{~mL}$ thrombus downstream; (b) $2 \mathrm{~mL}$ thrombus downstream. Wall shear stresses along the right and left walls of the vena cava are shown in the top and bottom figures, respectively. Gaps in the WSS profiles indicate the locations of renal inflow.

There are two competing flow processes that are balanced when the OF is minimized: the low WSS due to stagnant/recirculating flow between clots A and $\mathrm{B}$, and high WSS due to rapid flow through the narrow gap between clot $\mathrm{B}$ and the left wall of the cava. As clot A moves upstream, its wake occupies a larger portion of the cava flow between clots $\mathrm{A}$ and $\mathrm{B}$. This region of stagnant/recirculating flow yields a low WSS along the right cava wall, which increases the value of the OF. Conversely, as clot A moves downstream, the WSS along the left wall increases due to the large velocity gradients surrounding clot B. The large wall shear stresses that result increase the value of the OF. Consequently, there are flow factors that repel clot A from a far upstream location and from a location that is proximal to clot B. The optimal balance of these considerations determines the minimum of the $\mathrm{OF}$ and the optimal location of the upstream thrombus.

\subsection{Two parameter problem: Variable location and shape of the upstream thrombus}

The y-coordinate and the shape, as determined by the aspect ratio, of clot A are the design variables in the two parameter optimization problem. The clot once again remains confined to the cava wall, and the area of clot A remains constant. Normalized velocity contours for the optimal location and shape of the upstream thrombus are shown in Fig. 7a; the baseline configurations are shown in Fig. 5. Contour plots of the baseline velocities are the same as for the 
one parameter problem and are shown in Figs. 5a and 5b. As illustrated, the shape of clot $\mathrm{A}$ is streamlined with the flow, and the disruption to otherwise unoccluded IVC flow is confined to regions proximal to the thrombus; flow along the wall ipsilateral to the clot is unaltered. The size of clot B has minimal impact on the optimal aspect ratio of clot $\mathrm{A}$, and the small wake of the upstream thrombus does not interact with the flow dynamics that surround the downstream thrombus.
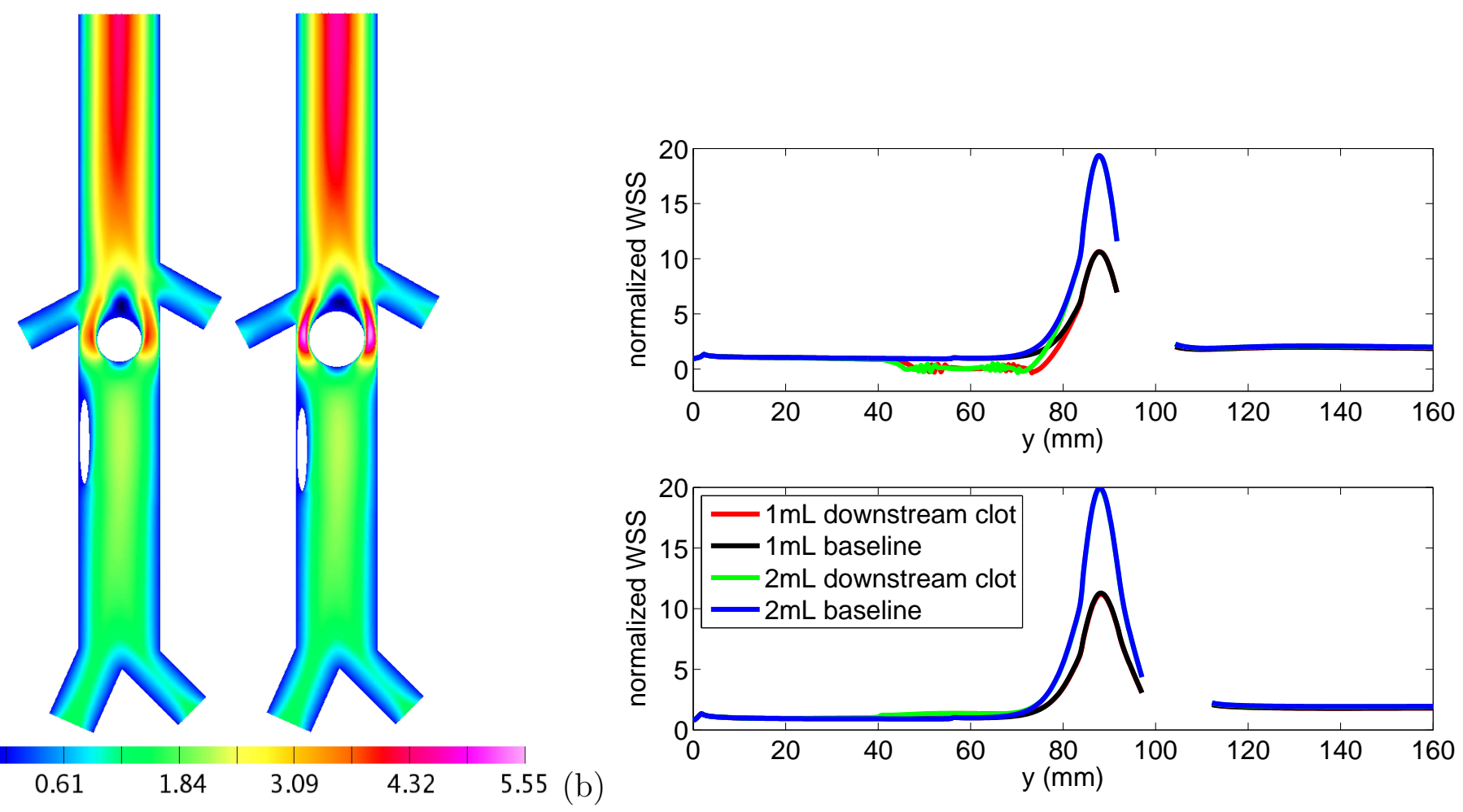

Fig. 7. Flow characteristics for the optimal configuration with two design parameter: (a) normalized y-component of velocity with $0.5 \mathrm{~mL}$ upstream thrombus and $1 \mathrm{~mL}$ (left) and $2 \mathrm{~mL}$ (right) downstream thrombus; (b) normalized WSS along the right (top) and left (bottom) walls of the vena cava. Gaps in the WSS profiles indicate the locations of renal inflow.

The normalized WSS profiles are shown in Fig. 7b. In all cases, the WSS profiles of the optimal configurations are virtually identical to the corresponding baseline case. The most notable differences lie in the vicinity of the upstream thrombus, where low wall shear stresses are observed beneath clot A. The magnitude of the wall shear stresses proximal to clot A are smaller than the corresponding values for circular thrombus (Fig. 6), but the fraction of vessel exposed to abnormal WSS is larger due to the elongated shape of the streamlined clot. Due to the streamlined shape of clot A and the large distance that separates clots $\mathrm{A}$ and $\mathrm{B}$, the fraction of the right cava wall that is exposed to low WSS is independent of the size of clot B. In short, streamlining the shape of the upstream clot reduces its influence on the flow dynamics and WSS. 
The trade-off that determines the optimal configuration of clot A is a balance between minimizing the wake effects by streamlining the shape and minimizing the fraction of the cava wall beneath the clot. Due to the constraint that clot A must remain along the right wall of the cava, these two competing processes are balanced when the $\mathrm{OF}$ is minimized. Unlike the one parameter problem described above, clot $\mathrm{B}$ plays a minimal role in determining the optimal configuration as apparent by the nearly identical solutions illustrated in Fig. 7a, where the volumes of clot B are $1 \mathrm{~mL}$ and $2 \mathrm{~mL}$. Note that including the aspect ratio of clot A as a design parameter allows the final WSS profile of the two parameter problem to better match the baseline configuration when compared to the corresponding one parameter problem (i.e., Fig. 6 versus Fig. 7b).

\section{Discussion}

Vena cava filters that trap thrombus along the wall of the IVC induce regions of stagnant/recirculating flow and abnormal WSS proximal to the thrombus. These areas of disrupted flow elevate the risk of thrombosis and should be minimized (e.g., [25-28]). One way to reduce the volume of stagnant/recirculating flow is to streamline the shape of all thrombi trapped along the wall, which reduces the vorticity downstream of the thrombus. But, when the shape of a thrombus is streamlined by stretching it along the axis of the cava, the area of cava wall beneath the thrombus increases, thereby exposing the wall to low WSS. The two parameter problem described above determines the optimal aspect ratio of the upstream thrombus (for a fixed size of clot) based on WSS considerations. As illustrated in Fig. 7a, the final shape balances the benefits of undisturbed flow downstream of the thrombus with the drawback of increasing the area of the IVC under the thrombus. Further specifying the shape of the upstream thrombus with additional design parameters may yield a more optimal shape, which gives a lower value of the OF.

In clinical applications, the optimal location and shape with which to trap thrombus is patient-specific (due to anatomic variance). Consequently, the optimal configuration for thrombus will change from patient-to-patient, and the results obtained here are strictly valid only under the idealized flow conditions considered. Hence, the ability of the present study to guide the design of practical devices is, in part, dependent on the sensitivity of the OF to perturbations of the flow conditions and thrombus morphology. To gain some insight into the impact of thrombus size on the optimal configuration, note from Table 3 that the solutions to the one and two parameter problems are relatively

insensitive to the size of thrombus. That is, doubling the effective volume of the upstream and downstream thrombus change the optimal locations and aspect ratios by less than $5 \%$ and $4 \%$, respectively. Although additional sensitivity studies are warranted, these results suggest that the design of a single 
Table 3

\begin{tabular}{|c|c|c|c|c|}
\hline \# design params & clot A $(\mathrm{mL})$ & clot B $(\mathrm{mL})$ & $y_{\text {opt }}(\mathrm{mm})$ & $a_{\text {opt }}$ \\
\hline \hline 1 & 0.5 & 1 & 72.04 & - \\
\hline 1 & 1 & 1 & 72.06 & - \\
\hline 1 & 0.5 & 2 & 71.50 & - \\
\hline 1 & 1 & 2 & 71.10 & - \\
\hline 2 & 0.5 & 1 & 60.22 & 8.76 \\
\hline 2 & 0.5 & 2 & 57.82 & 8.44 \\
\hline
\end{tabular}

Optimal location, $y_{\mathrm{opt}}$, and aspect ratio, $a_{\mathrm{opt}}$, of the upstream thrombus for all design optimization studies. The effective volumes of all upstream and downstream thrombi refers to the volume corresponding to the two-dimensional projections used in the current study.

filter may be near-optimal for a variety of applications.

Maximizing the efficacy of medical devices, including IVC filters, benefits from a systems design approach to modeling. In the present work, for example, the computational model and design criterion incorporate two thrombi and inflow from the common iliac and renal veins. As a result, the optimal location and shape of the upstream thrombus is influenced by a number of physiologically relevant flow processes (e.g., stagnant/recirculating flow, nonuniform flow into the IVC, interaction of downstream thrombus with renal inflow). Among the most notable flow interactions captured by the model is the coupling of the wake of clot A with the high speed flow between clot B and the cava wall. As clot A moves downstream during the design iteration process, flow in the wake of clot A interacts with the region of high pressure that is upstream of clot B; the high pressure region accelerates flow in the wake and reduces the area of stagnant/recirculating flow, which in turn increases the WSS downstream of clot A. At the same time, the WSS along the left cava wall is also monitored by the $\mathrm{OF}$ so as to mitigate any negative impacts to this region of the flow. It is interesting to note that, based on this work, the optimal separation distance between a $0.5 \mathrm{~mL}$ upstream thrombus and a $1.5 \mathrm{~mL}$ downstream thrombus is approximately $17 \mathrm{~mm}$ (results not shown). The corresponding separation distance of the TrapEase vena cava filter is approximately $34 \mathrm{~mm}$.

The present study includes a number of simplifying assumptions that reduce the computational expense and may be relaxed in future work. The use of a two-dimensional model, for example, reduces the number of grid points that are required to resolve the flow and therefore reduces the number of equations that must be solved when evaluating the OF. For the low Reynolds number, laminar flow considered here, a two-dimensional model is sufficient for understanding the overall flow dynamics. That is, changes to the location and 
shape of clot A modify the WSS upstream of the renal inflow, which alters the value of the OF; the WSS downstream of clot B is primarily determined by the renal inflow and remains virtually unaltered by clot $\mathrm{A}$. Hence, moving clot A along the cava wall upstream of clot B drives the system to an optimal design. In this region of the cava, however, the flow is predominately in the axial direction and therefore a two-dimensional model is reasonable.

The model assumes that all vessels are straight and rigid, all thrombus are circular or elliptical, and flow through the IVC is steady; the applicability of these approximations is discussed elsewhere (e.g., $[13,16])$. It can be noted, however, that the design optimization methodology used here is broadly applicable to different sizes and shapes of clots. As the diversity and complexity of clot morphology increases, the number of design parameters, $p$, required to characterize the clot also increases, which elevates the computational expense. Nonetheless, device designers may be able to exploit these additional degrees of freedom when constructing new devices.

\section{Conclusions}

A design optimization framework that couples methods of computational fluid dynamics and nonlinear optimization has been constructed and applied to problems in venous thrombosis. The flow solver computes solutions to the incompressible Navier-Stokes equations, and the optimization solver minimizes a user-specified objective function. A fully automated coupling of the two solvers is implemented, tested, and the results are verified on three test problems.

The computational framework is used to determine the optimal configuration of thrombus that may be trapped by vena cava filters. It is found that thrombus in close proximity can leverage flow characteristics and mitigate the risk of thrombosis. In addition, streamlined thrombi trapped against the cava wall minimize disruption to the flow and wall shear stress.

The modeling capabilities described here are robust and broadly applicable. Future work may include extension to three-dimensions, incorporation of additional design parameters, and the formulation and application of more inclusive objective functions. Further, the framework may be applied to other medical devices. 


\section{Acknowledgements}

LLNL is operated by Lawrence Livermore National Security, LLC, for the DOE, National Nuclear Security Administration under Contract DE-AC52$07 N A 27344$.

\section{References}

[1] Mackman, N., 2008, "Triggers, Targets and Treatments for Thrombosis," Nature, 451(21), pp. 914-918.

[2] Wakefield, T.W., Caprini, J., and Comerota, A.J., 2008, "Thromboembolic Disease," Curr. Probl. Surg., 45, pp. 844-899.

[3] Heit, J.A., Cohen, A.T., and Anderson, F.J., 2005, "Estimated Annual Number of Incident and Recurrent, Non-Fatal Venous Thromboembolism (VTE) Events in the U.S.," Blood, 106: Abstract 910.

[4] Lee, P., Raizada, A., Ciocca, R., 2009, "Growing Utilization of IVC Filter Placement from 2001-2005: Analysis of NIS," Abstract for the Society for Clinical Vascular Surgery 2009 Meeting, Accessed January 22, 2009.

[5] Kroll, M.H., Hellums, J.D., Mcintire, L.V., Schafer, A.I., and Moake, J.L., 1996, "Platelets and Shear Stress," Blood, 88, pp. 15251541.

[6] Lowe, G., 2003/2004, "Virchow's Triad Revisited: Abnormal Flow," Pathophysiol. Haemost. Thromb., 33, pp. 455457.

[7] Marsden, A.L., Feinstein, J.A., and Taylor, C.A., 2008, "A Computational Framework for Derivative-Free Optimization of Cardiovascular Geometries," Comput. Methods Appl. Mech. Engrg., 197, pp. 1890-1905.

[8] Pekkan, K., Whited, B., Kanter, K., Sharma, S., de Zelicourt, D., Sundareswaran, K., Frakes, D., Rossignac, J., Yoganathan, A.P., 2008, "PatientSpecific Surgical Planning and Hemodynamic Computational Fluid Dynamics Optimization through Free-Form Haptic Anatomy Editing Tool (SURGEM)", Med. Biol. Eng. Comput., 46(11), pp. 1139-52.

[9] Timmins, L.H., Moreno, M.R., Meyer, C.A., Criscione, J.C., Rachev, A., and Moore Jr., J.E., 2007, "Stented Artery Biomechanics and Device Design Optimization," Med. Bio. Eng. Comput., 45, pp. 505-513.

[10] De Beule, M., Van Cauter, S., Mortier, P., Van Loo, D., Van Impe, R., Verdonck, P., and Verhegghe, B., 2009, "Virtual Optimization of Self-Expandable Braided Wire Stents," Med. Eng. Phys, 31(4), pp. 448-453.

[11] Srinivas, K., Nakayama, T., Ohta, M., Obayashi, S., and Yamaguchi, T., 2008, "Studies on Design Optimization of Coronary Stents," J. Med. Devices, 2(1), 011004 . 
[12] Stewart, S.F.C., Robinson, R.A., Nelson, R.A., and Malinauskas, R.A., 2008, "Effects of Thrombosed Vena Cava Filters on Blood Flow: Flow Visualization and Numerical Modeling," Ann. Biomed. Eng., 36(11), pp. 1764-1781.

[13] Singer, M.A., Henshaw, W.D., and Wang, S.L., 2009 "Computational Modeling of Blood Flow in the TrapEase Inferior Vena Cava Filter," J. Vasc. Interv. Radiol., 20, pp. 799-805.

[14] Wang, S.L. and Singer, M.A., "Toward an Optimal Position for IVC Filters: Computational Modeling of the Impact of Renal Vein Inflow," J. Vasc. Interv. Radiol., in press.

[15] Singer, M.A. and Wang, S.L., "Modeling Flow Past a Tilted Vena Cava Filter," J. Biomed. Eng., submitted.

[16] Swaminathan, T.N., Hu, H.H., and Patel, A.A., 2006, "Numerical Analysis of the Hemodynamics and Embolus Capture of a Greenfield Vena Cava Filter," J. Biomed. Eng., 128, pp. 360-370.

[17] Kaufman, J.A., Waltman, A.C., Rivitz, S.M., and Geller, S.C., 1995, "Anatomical Observations on the Renal Veins and Inferior Vena Cava at Magnetic Resonance Angiography," Cardiovasc. Interv. Radiol., 18, pp. 153157.

[18] Wang, S.L., Timmermans, H.A., and Kaufman, J.A., 2007, "Estimation of Trapped Thrombus Volumes in Retrievable Inferior Vena Cava Filters: A Visual Scale," J. Vasc. Interv. Radiol., 18, pp. 273-276.

[19] Chesshire, G.S. and Henshaw, W.D., 1990, "Composite Overlapping Meshes for the Solution of Partial Differential Equations," J. Comp. Phys., 90(1), pp. 1-64.

[20] Henshaw, W.D., 1994. "A Fourth-Order Accurate Method for the Incompressible Navier-Stokes Equations on Overlapping Grids," J. Comp. Phys., 113(1), pp. 13-25.

[21] Henshaw, W.D. and Petersson, N.A., 2003, "A Split-Step Scheme for the Incompressible Navier-Stokes Equations," In: Hafez MM, (Ed.), Numerical Simulation of Incompressible Flow, World Scientific, pp. 108-125.

[22] Gray, G.A. and Kolda, T.G., 2006, "Algorithm 856: APPSPACK 4.0: Asynchronous Parallel Pattern Search for Derivative-Free Optimization," ACM Trans. Math. Softw., 32(3), pp. 485-507.

[23] Kolda, T.G., 2005, "Revisiting Asynchronous Parallel Pattern Search for Nonlinear Optimization," SIAM J. Optim, 16(2), pp. 563-586.

[24] Gray, G.A., Kolda, T.G., Sale, K.L., and Young, M.M., 2004, "Optimizing an Empirical Scoring Function for Transmembrane Protein Structure Determination," INFORMS Journal on Computing, 16(4), pp. 406-418.

[25] Leask, R.L., Johnston, K.W., and Ojha, M., 2004, "Hemodynamic Effects of Clot Entrapment in the TrapEase Inferior Vena Cava Filter," J. Vasc. Interv. Radiol., 15, pp. 485-490. 
[26] Harlal, A., Ojha, M., and Johnston, K.W., 2007, "Vena Cava Filter Performance Based on Hemodynamics and Reported Thrombosis and Pulmonary Embolism Patterns," J. Vasc. Interv. Radiol., 18, pp. 103-115.

[27] Dintenfass, L., 1964, "Rheological Approach to Thrombosis and Atherosclerosis," Angiology, 15, pp. 333-343.

[28] Schoephoerster, R.T., Oynes, F., Nunez, G., Kapadvanjwala, M., and Dewanjee, M.K., 1993, "Effects of Local Geometry and Fluid Dynamics on Regional Platelet Deposit on Artificial Surfaces," Arterioscler. Thromb., 13, pp. 18061813.

[29] Rahbar, E., Moli, D., and Moore, J., 2009, "Three-Dimensional Analysis of Flow Disturbances from Clots in Vena Cava Filters," J. Vasc. Interv. Radiol., 20(2), pp. S22.

[30] Cheng, C.P., Herfkens, R.J., and Taylor, C.A., 2003, "Inferior Vena Caval Hemodynamics Quantified In Vivo at Rest and During Cycling Exercise Using Magnetic Resonance Imaging," Am. J. Physiol. Heart Circ. Physiol., 284, pp. 1161-1167.

[31] Roache, P.J., 1997, "Quantification of Uncertainty in Computational Fluid Dynamics," Annu. Rev. Fluid Mech., 29, pp. 123-160.

\section{Conflict of interest statement}

The authors declare no conflicts of interest. 